\title{
The effect of hydroxyethyl starches and crystalloid fluids on mortality and need for dialysis in vascular surgery patients
}

\author{
R Green ${ }^{1,2,3^{*}}$, S Hicks' ${ }^{2}$ M Butler ${ }^{1,2,3}$ \\ From ESICM LIVES 2015 \\ Berlin, Germany. 3-7 October 2015
}

\begin{abstract}
Introduction
Previous research has indicated that fluid choice is an important component of the resuscitation of the critically-ill patient. Synthetic hydroxyethyl starches (HES) may remain within the intravascular space compared to crystalloid solutions, yet they have also been associated with acute kidney injury. Data comparing HES and crystalloid solutions in anesthesia is incomplete. The goal of this study was to evaluate the effect of utilizing HES on adverse patient outcomes in vascular surgery patients at a Canadian academic hospital.
\end{abstract}

\section{Objectives}

To compare the association of the administration of HES solutions with crystalloid solutions on in-hospital mortality and the requirement for hemodialysis in patients that underwent a vascular surgery procedure.

\section{Methods}

This was an unblinded, retrospective review of 796 patients that underwent a vascular surgery procedure between 2007 and 2009. The patients were divided into those that received HES intraoperatively $(n=213)$ and those that received crystalloid fluids $(n=583)$. The data were abstracted from an electronic intraoperative information system, a hospital central patient registry, and the patient record. Inclusion criteria were all adult $(\geq 16$ years) patients that were intubated for a vascular surgery procedure.

To assess for an association between in-hospital mortality, hemodialysis requirement, ventilator requirement and inotrope requirement we utilized a logistic regression

${ }^{1}$ Dalhousie University, Nova Scotia Truama Program, Halifax, Canada Full list of author information is available at the end of the article model that adjusted for gender, age, urgency of procedure and American Society of Anesthesiologists (ASA) score.

\section{Results}

Receiving HES solutions was associated with a greater post-operative hemodialysis requirement $(\mathrm{OR}=19.53$, CI: $1.54,247.0)$, inotrope requirement $(\mathrm{OR}=2.74$, CI: $1.48,5.08)$, ventilator requirement $(\mathrm{OR}=8.50, \mathrm{CI}: 5.40$, 13.40), and intensive care unit (ICU) admission (OR = 9.81, CI: 6.51, 14.77). Patients that received HES solutions were more likely to be admitted to both an inpatient ward and an ICU. Receiving HES solutions was not associated with in-hospital mortality $(\mathrm{OR}=1.50$, CI: $0.77,2.91)$.

\section{Conclusions}

In our study of vascular surgery patients of varying acuity, administration of HES was associated with poor patient outcomes compared to crystalloid solutions. More study is required to fully elucidate the effects of HES on in-hospital mortality and hemodialysis requirements of this patient population.

\section{Authors' details}

'Dalhousie University, Nova Scotia Truama Program, Halifax, Canada. ${ }^{2}$ Dalhousie University, Department of Critical Care, Halifax, Canada. ${ }^{3}$ Dalhousie University, Department of Emergency Medicine, Halifax, Canada.

Published: 1 October 2015

doi:10.1186/2197-425X-3-S1-A744

Cite this article as: Green et al:: The effect of hydroxyethyl starches and crystalloid fluids on mortality and need for dialysis in vascular surgery patients. Intensive Care Medicine Experimental 2015 3(Suppl 1):A744.

\section{SpringerOpen ${ }^{\odot}$}

( 2015 Green et al.; This is an Open Access article distributed under the terms of the Creative Commons Attribution License (http:// creativecommons.org/licenses/by/4.0), which permits unrestricted use, distribution, and reproduction in any medium, provided the original work is properly cited. 